\title{
neofilolog
}

Czasopismo Polskiego Towarzystwa Neofilologicznego

ISSN 1429-2173, elSSN 2545-3971, 2021, NR 56/2, 169-184

http://dx.doi.org/10.14746/n.2021.56.2.2

http://poltowneo.org/

Beata Gałan

Uniwersytet Śląski

https://orcid.org/0000-0002-9244-448X

beata.galan@us.edu.pl

\section{La classe de langue à l'ère du digital. Quelques réflexions sur l'intégration des compétences numériques en didactique des langues étrangères}

\section{The language class in the digital age. Towards the integration of digital skills in foreign language teaching}

Digital skills are an important part of the skills required in the 21st century, enabling users to better utilise information and communication technologies (ICT) in different spheres of life. This article examines the integration of these skills into foreign language teaching. The first part of the article studies the notion of digital skills through initiatives undertaken at the European and the national level as well as the current role of digital technology in the language classroom. The second part of the article focuses on the possible integration of digital skills with the development of productive language skills through active methods (collaborative learning, project-based learning, problem-based learning). The preliminary examination shows that the use of these teaching strategies could possibly ensure the parallel development of language and digital skill.

Keywords: digital skills, language class, information and communications technologies, activating methods

Słowa kluczowe: kompetencje cyfrowe, klasa językowa, technologie informacyjne i komunikacyjne, metody aktywizujące 


\section{Introduction}

Cet article a pour objectif d'interroger la possibilité d'intégrer les compétences numériques dans le processus d'enseignement-apprentissage des langues étrangères. Avec la digitalisation progressive de la société et les nombreux changements qui en résultent, les compétences numériques font désormais partie des compétences indispensables dans la société du XXI siècle. L'importance accordée à leur développement est soulignée dans différents programmes et initiatives entrepris à l'échelle mondiale, européenne et nationale. Figurant parmi les huit compétences clés mises en avant par la stratégie européenne pour l'éducation et la formation tout au long de la vie (Commission européenne, 2006, 2018), ces compétences « impliqu[ent] l'usage sûr et critique des technologies de la société de l'information (TSI) au travail, dans les loisirs et dans la communication » (Commission européenne, $2006: 6$ ) pour accorder aux usagers une meilleure compréhension et gestion des opportunités offertes par les technologies de l'information et de la communication (TIC) dans différentes sphères de la vie sociale et éducative.

L'intégration des compétences numériques dans la formation linguistique nous importe pour deux raisons. D'un côté, le recours aux compétences numériques optimiserait le processus d'enseignement-apprentissage qui demeure de plus en plus souvent soutenu par la technologie numérique. De l'autre côté, la formation linguistique contribuerait au développement des savoirs et des savoir-faire facilitant un meilleur fonctionnement dans la société numérique. La présente contribution analysera ces opportunités : la première partie de l'article évoquera la notion de compétences numériques et le rôle de la technologie numérique en classe de langue ; la seconde partie proposera quelques réflexions théoriques sur l'intégration de ces premières dans le développement des compétences langagières, notamment celles qui nécessitent la production et l'interaction.

\section{Les compétences numériques}

Les compétences numériques se développent dans différents domaines conformément à la révolution digitale. De plus, elles font l'objet de plusieurs programmes et initiatives élaborées à l'échelle mondiale comme : Framework for 21st Century Learning et Future Work Skills 2020, européenne - Stratégie Europe 2020, Le M anifeste pour les compétences numériques (2019) ou nationale - PO Polska Cyfrowa (Programme Opérationnel Pologne Numérique), Cyfrowa Szkoła (École Numérique), Szkoła dla Innowatora (École pour Innovateur). Toutes ces initiatives se construisent autour de la notion de compétences 
numériques qui fonctionnent également sous le terme de capacités/ habiletés numériques, e-compétences (digital skills, e-skills), compétences médiales ou compétences informationnelles. La notion, si bien ancrée dans le discours politique, professionnel et éducatif, présente néanmoins une forte hétérogénéité dans la définition (Mell, 2018). D’une manière générale, les compétences numériques renvoient à « la connaissance approfondie et à la capacité à apprendre toute sa vie durant dans tous les domaines de la révolution numérique, ses technologies, ses applications, ses utilisations et les possibilités de transformation radicale qu'elle offre » (Commission européenne, 2012 : 9). M ême si les capacités techniques ${ }^{1}$ constituent une condition préalable pour le développement des dites compétences, celles-ci pourraient également être qualifiées de nature sociale et comportementale (Bouillon, 2015) étant donné qu'elles impliquent le travail collaboratif ou encore l'aptitude individuelle à interagir avec le numérique. Ainsi, les compétences numériques renvoient, conformément à la notion de compétence, aux connaissances dites déclaratives, procédurales mais également aux compétences comportementales de l'individu (savoir-être) correspondant entre autres aux attitudes et valeurs (Vienneau, 2011 : 20). Vu le nombre de facteurs qu'évoquent les compétences numériques, il serait difficile de cerner toute cette complexité dans le cadre restreint d'un article. Néanmoins, afin de mieux saisir cette notion, prenons deux projets traitant le développement de compétences numériques à l'échelle européenne et nationale : le référentiel européen DIGCOMP et le Programme Opérationnel Pologne Numérique (Polska Cyfrowa), élaborés dans le cadre de la Stratégie Europe 2020.

Le DIGCOM P, publié pour la première fois en 2013, est un cadre de référence pour les compétences numériques, élaboré dans la continuité des initiatives européennes en matière d'éducation et la formation tout au long de la vie (Commission européenne, 2018). D'un point de vue général, le cadre comprend les compétences numériques en termes de catalogue regroupant différentes connaissances et capacités en matière de TIC tout en soulignant différents domaines d'activité dans lesquels celles-ci pourraient se manifester. La dernière version du cadre, signée 2.1 , distingue 21 compétences numériques regroupées en cinq domaines (Carretero S., Vuorikari R., Punie Y., 2017 : 21) :

${ }^{1}$ P.ex. la maîtrise de l'outil informatique. 


\begin{tabular}{|c|c|}
\hline Domaine d'activité & Compétences \\
\hline Informations et données & $\begin{array}{l}\text { 1.1 Mener une recherche et une veille d'information } \\
\text { 1.2 Gérer des données } \\
\text { 1.3 Traiter des données }\end{array}$ \\
\hline \multirow{6}{*}{$\begin{array}{l}\text { Communication et collabo- } \\
\text { ration }\end{array}$} & 2.1 Interagir \\
\hline & 2.2 Partager et publier \\
\hline & 2.3 Collaborer \\
\hline & 2.4 S'insérer dans le monde numérique \\
\hline & 2.5 Nétiquette \\
\hline & 2.6 Gérer l'identité numérique \\
\hline \multirow[t]{4}{*}{ Création de contenus } & 3.1 Développer des documents textuels \\
\hline & 3.2. Développer des documents multimédia \\
\hline & 3.3 Adapter les documents à leur finalité \\
\hline & 3.4 Programmer \\
\hline \multirow[t]{4}{*}{ Protection et sécurité } & 4.1 Sécuriser l'environnement numérique \\
\hline & 4.2 Protéger les données personnelles et la vie privée \\
\hline & 4.3 Protéger la santé, le bien-être \\
\hline & 4.4. Protéger l'environnement \\
\hline \multirow[t]{4}{*}{ Environnement numérique } & 5.1 Résoudre des problèmes techniques \\
\hline & 5.2 Évoluer dans un environnement numérique \\
\hline & $\begin{array}{l}5.3 \text { Utiliser les technologies numériques de manière } \\
\text { créative }\end{array}$ \\
\hline & 5.4 Identifier les lacunes de compétences numé- \\
\hline
\end{tabular}

Tab 1. Source : Carretero S., Vuorikari R., Punie Y., (2017 : 21) ; M inistère de l'Education nationale et de la Jeunesse (2019: 7).

En partant du principe que les compétences numériques s'acquièrent au cours de la formation formelle ou informelle, le cadre DIGCOMP propose quatre principaux niveaux de maîtrise (novice, indépendant, avancé, expert) pour évaluer ou autoévaluer les compétences en question ${ }^{2}$. Ce faisant, les compétences numériques sont comprises en tant que résultat de la performance de l'usager face à la réalisation d'une tâche dans le milieu numérique. Autrement dit, elles correspondent, comme chaque autre compétence, à une entité cognitive et sociale, liée d'une manière intrinsèque au jugement social (Coulet 2011 : 4). Sur le terrain polonais, l'inspiration du cadre DIGCOM P se retrouve dans le catalogue des compétences médiatiques, informationnelles et numériques, proposé dans le cadre du projet Futur Numérique (Cyfrowa Przyszłość) de La Fondation Pologne M oderne (Fundacja Nowoczesna Polska).

${ }^{2}$ Dans le contexte français, le développement des compétences numériques s'inscrit dans le parcours scolaire et propose une certification, inspirée du cadre DIGCOM P, en fin de cycle 4 du collège et au cycle terminal du lycée (M inistère de l'Education nationale et de la Jeunesse, 2019). 
La classe de langue à l'ère du digital. Quelques réflexions sur l'intégration...

Ce projet promeut l'éducation aux médias dans l'enseignement formel et informel (Budzisz et al., 2014).

Une autre approche est présentée dans le programme la Pologne Numérique (Polska Cyfrowa), conçu pour les années 2014-2020. Les auteurs du programme proposent un modèle relationnel des compétences numériques sans l'envisager comme un champ d'activité à part. Ils le pensent davantage comme " un ensemble harmonieux des connaissances, des capacités et attitudes qui permettent d'utiliser les technologies numériques de manière efficace dans différentes sphères de notre vie » (Buchholtz et al, $2015: 14)^{3}$. Le modèle en question suppose que le développement des compétences numériques s'effectue individuellement pour chaque usager et en fonction de ses besoins dans différents domaines d'activité ${ }^{4}$. Bien que cette conception souligne certaines lacunes de l'approche dite normative proposée par le cadre DIGCOM P, elle complète et souligne le caractère dynamique et individuel des compétences numériques.

Dans le modèle relationnel, les compétences numériques sont classifiées comme fonctionnelles - leur acquisition et développement ne constituent pas un objectif en soi, mais réalisent différentes tâches par le biais de la technologie numérique. Ainsi, les compétences fonctionnelles englobent les compétences fondamentales en usage du numérique, à savoir :

1. informatiques - connaissances concernant l'utilisation de l'ordinateur (ou d'autres appareils électroniques), d'Internet, des logiciels et d'applications ainsi que les connaissances liées à la création du contenu numérique,

2. informationnelles - connaissances relatives à la recherche de l'information et à la gestion des ressources numériques (Jasiewicz et al., 2015: 6-8).

Selon les concepteurs du modèle, ces capacités constituent une base pour le développement des compétences numériques, à laquelle s'ajoutent d'autres qui tiennent au parcours individuel de l'usager. De cette manière, chaque individu

3 „Przyjmujemy zatem, że e-kompetencje to harmonijny zespół wiedzy, umiejętności i postaw, które pozwalają efektywnie wykorzystywać technologie cyfrowe w różnych obszarach życia." (Buchholtz et al., 2015 : 14)

${ }^{4}$ La recherche réalisée par le Centre Numérique en Pologne (Centrum Cyfrowe. Projekt Polska) (Filiciak et al., 2013) a distingué huit principaux domaines d'activité des Polonais : la vie quotidienne, les finances, les relations interpersonnelles, le travail et le développement professionnel, l'engagement citoyen, la santé, les loisirs et la religion (Jasiewicz et al., $2015: 9$ ) 
construit son propre catalogue de compétences numériques modelé par son environnement, sa situation professionnelle et ses motivations.

M ême si les deux approches présentées ne donnent qu'un aperçu, elles proposent une conception complémentaire des compétences numériques, pouvant servir de référence. Considérant cette complémentarité, les compétences numériques pourraient se comprendre en termes de connaissances et aptitudes qui forment « un ensemble évolutif de capacités cognitives, de dispositions et de savoir-faire individuels appliqué aux technologies numériques » (M ell, 2018 : 177). Afin d'offrir aux apprenants un cadre favorable pour une progression cohérente en compétences numériques, nous estimons indispensable d'assurer l'intégration optimale de la technologie numérique dans différents champs éducatifs.

\section{La technologie numérique en classe de langue}

Le recours à la technologie numérique dans le milieu éducatif peut prendre différentes formes. Selon Puentedura (2006), quatre principaux niveaux de cette intégration se distinguent : la substitution ( $S$ ) où la technologie ne fait que remplacer l'outil traditionnel ; I'augmentation (A) qui privilégie l'usage fonctionnel de la technologie ; la modification ( $M$ ) où le numérique entraîne une transformation significative de la tâche et la redéfinition (R) qui autorise une nouvelle conception de la tâche impossible à réaliser antérieurement (Lebrun, Lison, Batier, 2016 : 2-3). Conformément à ce modèle, la présence du numérique dans l'activité didactique varie d'un simple usage accentuant la forme plutôt transmissive de l'enseignement à l'usage plus créatif, favorisant le caractère interactif et participatif des technologies de l'information et de la communication. Actuellement, on dispose de différents outils et ressources numériques - éducatifs et de grand public, disponibles en et hors-ligne. Citons entre autres les applications Internet et mobiles, les ressources visuelles et audiovisuelles, les sites éducatifs ou les systèmes logiciels de gestion d'enseignement. Ces outils offrent des exploitations variées dans le processus d'enseignement-apprentissage des langues étrangères pour le développement des compétences réceptives, mais aussi productives qui nécessitent la mise en place des activités assurant l'expression et l'interaction orale et écrite. Certains outils (p.ex. les plateformes d'enseignement à distance) offrent également des conditions d'apprentissage plus adaptées aux contraintes géographiques et temporelles que la classe réelle. En se référant aux environnements interactifs en ligne, il est possible de mettre en œuvre l'apprentissage dit hybride, reposant sur le concept blended -learning (Mangenot, 2011) et proposant la combinaison des pratiques d'apprentissage en présentiel et en 
La classe de langue à l'ère du digital. Quelques réflexions sur l'intégration...

distanciel (Nissen, 2019) ou encore l'apprentissage à distance, s'inscrivant dans les pratiques de l'e-learning (Rossenberg, 2000 ; Glikman, 2002) et aidant à numériser tous les échanges dans l'espace virtuel.

Considérant toutes ces possibilités, il importe de souligner que le caractère de l'usage de la technologie en classe dépend de plusieurs critères. Dans une certaine mesure, ce caractère est déterminé déjà à l'étape du choix de l'outil qui permet/ne permet pas l'application de certaines pratiques. Néanmoins, ce n'est pas la présence en soi de la technologie en classe qui modifie l'apprentissage mais la façon dont celui-ci intègre l'activité didactique (Bachy, 2019 : 127). Malgré la démocratisation des dispositifs digitaux, nous pouvons observer que le potentiel des TIC en matière d'apprentissage n'est que partiellement exploité par les enseignants et les apprenants. En analysant les pratiques de l'usage du numérique dans le contexte éducatif, on constate que les enseignants recourent à la technologie généralement pour remplacer l'outil traditionnel (Plebańska, Szyller, Sieńczawska, 2020 : 133-153), tout en limitant le caractère interactif et participatif des outils numériques (Ollivier et Puren, 2011). Cet usage élémentaire du numérique ne correspond pas auxattentes des apprenants, considérés comme digital natives (Prensky, 2001) qui privilégient les tâches créatives et l'apprentissage multimodal (żylińska, 2013 ; Nowicka et Dziekońska, 2018). De l'autre côté, même si les apprenants se caractérisent par une attitude plutôt technophile, ils présentent quelques lacunes en termes de savoir-faire technique et conceptuel qui supporterait un usage plus raisonné de la technologie dans le contexte scolaire (Dauphin, 2012). En prenant en considération toutes ces observations, on pourrait constater que le numérique en classe de langue (tout comme dans le milieu éducatif en général) nécessite une intégration fondée sur les compétences relatives à l'usage conscient de la technologie qui offrirait aux apprenants et aux enseignants une meilleure exploitation du numérique au service de l'apprentissage.

\section{Vers l'intégration des compétences numériques en classe de langue}

Étant donné l'importance des compétences numériques, interrogeons leur intégration dans la classe de langue virtuelle, ayant lieu à distance et dans l'environnement médiatisé. Nous nous concentrerons plus précisément sur les compétences numériques qui, d'après le cadre DIGCOMP correspondent à trois domaines : la communication et collaboration, la création de contenus et le travail dans l'environnement numérique (Carretero S., Vuorikari R., Punie Y., 2017 : 21). Même si le développement parallèle des compétences langagières et numériques pourrait s'effectuer dans différents contextes didactiques, nous voudrions accentuer les approches pédagogiques ou stratégies d'enseignement qui : 
1) assureraient l'entraînement des compétences langagières en matière de production et d'interaction,

2) engageraient les compétences numériques des apprenants travaillant à distance et dans l'environnement numérique,

3) offriraient un usage créatif du numérique en classe de langue.

Dans la section suivante de l'article, nous nous demanderons si les approches actives, centrées sur l'apprenant, en l'occurrence, I'apprentissage collaboratif, l'apprentissage par projet et l'apprentissage par problème pourraient réaliser ces objectifs et donner une meilleure exploitation des TIC dans le processus d'enseignement-apprentissage des langues étrangères.

\subsection{L’apprentissage collaboratif, apprendre à collaborer et à communiquer en ligne}

Les compétences numériques en matière de communication et de collaboration englobent les habiletés telles que l'interaction, le partage, la publication, la collaboration, l'insertion dans le monde numérique, l'utilisation de la nétiquette et la gestion de l'identité numérique (Carretero S., Vuorikari R., Punie Y., 2017 : 21). Vu leur spécificité, ces compétences pourraient être mobilisées entre autres dans l'apprentissage collaboratif qui, réalisé dans l'environnement numérique, canalise divers modes de collaboration et de communication. Rappelons que l'apprentissage collaboratif est une "stratégie d'enseignement par laquelle un certain nombre d'élèves sont regroupés pour réaliser une tâche scolaire » (Vienneau, 2011 : 190). Cette démarche prend en considération la dimension individuelle et celle du groupe, autrement dit, elle « reconnaît le caractère individuel et réflexif de l'apprentissage de même que son ancrage social en le raccrochant aux interactions de groupe » (Henri, Lundgren-Cayrol, 2001 : 42). Dans l'espace virtuel, la collaboration aurait lieu dans une communauté d'apprentissage en ligne, sur une plateforme de socialisation ou sur une plateforme d'apprentissage. De principe, les deux premières se construisent particulièrement dans un contexte extrascolaire : la communauté d'apprentissage se forme de manière spontanée, autour d'un objectif commun, un intérêt partagé et sur une base volontaire (Henri 2010 : 173) tandis que la plateforme de socialisation se limite à un cercle d'amis de la vie réelle souhaitant échanger dans un espace virtuel. La plateforme d'apprentissage est mise en place dans l'apprentissage plus formel et réunit les apprenants inscrits en formation ainsi que l'équipe pédagogique. Les trois dispositifs regroupent différents outils pour maintenir la communication qui peut être synchrone (en temps réel) ou asynchrone (en temps différé), individuelle ou collective et utiliser différentes modalités (écrit, audio, vidéo). 
La classe de langue à l'ère du digital. Quelques réflexions sur l'intégration...

Même si certains outils de communication se caractérisent par une seule modalité (p.ex. le courriel électronique, le forum de discussion), beaucoup de ces outils en combinent plusieurs. Sous condition d'assurer un cadre technique satisfaisant, les outils de communication actuels garantissent le même type d'interaction qui a lieu en présentiel. Ceci facilite la démarche du groupe qui dans l'apprentissage collaboratif est une source d'information, de motivation et d'entraide (Henri, Lundgren-Cayrol, 2001 : 42). Afin de consolider l'espace nécessaire de collaboration, plusieurs outils peuvent être mis en place:

- les outils de collaboration textuels (p.ex. le wiki et le blogue) pour élaborer et publier des travaux écrits rédigés à plusieurs mains,

- les sites de partage et l'informatique en nuage dotés d'un espace suffisant pour emmagasiner et échanger les fiches de travail à différentes étapes de leur élaboration,

- les portfolios numériques pour archiver les productions d'apprentissage (travaux écrits, vidéos, enregistrements, présentations multimédias),

- les agendas numériques pour gérer le temps et optimiser le travail du groupe.

Tous ces outils peuvent être combinés de plusieurs manières et complétés par des dispositifs et applications extérieurs. En fonction des besoins, il est aussi possible de mettre en place différentes configurations de groupe : équipe de plusieurs personnes, petits groupes ou binômes. Grâce à ces possibilités, la collaboration en ligne peut prendre des formules comparables à son équivalent en présentiel tout en apportant des bénéfices supplémentaires, comme entre autres la flexibilité du temps et l'autonomie, la convivialité ou la capacité de supervision (Walckiers, De Preatere, 2004 : 62-67).

En prenant en considération ces éléments, on pourrait constater que l'apprentissage collaboratif en ligne peut procurer les conditions nécessaires pour coupler le développement des compétences langagières qui nécessitent la production et l'interaction orale et/ou écrite et des compétences numériques, notamment celles relatives à la communication et à la collaboration. En travaillant en ligne, les apprenants auraient l'occasion de s'insérer dans le monde numérique pour collaborer, ce qui exige la mise en place non seulement des habiletés de nature technique (comme la maîtrise du matériel et des outils) mais aussi sociale, permettant de renforcer une collaboration fondée sur la responsabilité et le respect des règles de bonne conduite dans l'espace virtuel. 


\subsection{L'apprentissage par projet en ligne et la création des contenus}

L'apprentissage par projet est souvent considéré comme une approche assurant le développement des compétences disciplinaires, mais aussi transversales (Vienneau, 2011 : 200). Ainsi, la mise en place de l'apprentissage par projet dans l'environnement médiatisé mobiliserait les compétences numériques, entre autres celles concernant la création de contenus, dont l'utilité se manifeste à travers les disciplines. II s'agirait alors des capacités à développer des documents textuels, développer des documents multimédia, adapter les documents à leur finalité et programmer (Carretero S., Vuorikari R., Punie Y., 2017 : 21). Dans ce contexte, il importe de souligner que, la démarche par projet est « une approche pédagogique qui pousse l'élève à s'engager pleinement dans la construction de ses savoirs en interaction avec ses pairs et son environnement » (Arpin, Capra, 2001 : 7). Elle s'appuie aussi sur le traitement de l'information, l'apprentissage stratégique et l'enseignement par médiation (Proulx, 2004 : 19) en soulignant tout particulièrement la participation active de l'apprenant durant toutes les étapes du projet. De ce fait, l'apprentissage par projet offre des conditions intéressantes pour mettre en place à la fois le développement des compétences langagières qui nécessitent la production et l'interaction et celles relatives à la création du contenu numérique. De principe, les activités (tâches) réalisées dans le cadre du projet doivent aboutir à un résultat final - une action concrète, un produit tangible ou une solution au problème. Dans le processus d'enseignement-apprentissage des langues étrangères, le projet consiste d'habitude à réaliser une production concrète. M ême si la démarche impose d'habitude une production langagière dominante (orale ou écrite), elle permet aussi d'intégrer différents types d'activités productives et réceptives.

L'apprentissage par projet soutenu par le numérique et appliqué au sein du processus d'enseignement-apprentissage des langues étrangères correspondrait entre autres à la réalisation des tâches médiatisées appelées également cybertâches ou missions virtuelles (M angenot, Soubrié, 2010). Dans cette optique et en fonction des objectifs à atteindre, le recours au numérique peut prendre différentes formes. Premièrement, les activités du projet consisteraient à consulter les documents supports (authentiques et/ ou didactisés) disponibles sur Internet. Deuxièmement, l'élaboration de la production exigerait la mise en place des outils qui permettent la création des contenus textuels et multimédias (les outils/applications de bureautique ou des logiciels/applications graphique, d'enregistrement audio, de montage de vidéo). Troisièmement, le projet s'appuierait sur la collaboration et la coopération nécessitant le recours aux outils assurant la collaboration et la communication à distance. Conformément à ces possibilités, on pourrait relever que la réalisation 
La classe de langue à l'ère du digital. Quelques réflexions sur l'intégration...

du projet dans l'environnement numérique offre un cadre propice au développement des performances linguistiques et des compétences numériques des apprenants. En premier lieu, les moyens technologiques actuels soutiendraient la création des travaux multimédias nécessitant la production et l'interaction, tels que les vidéos, les présentations multimédias interactives, les enregistrements audio, l'écriture collaborative, etc. Le travail avec les documents authentiques disponibles en ligne dans le cadre du projet favoriserait la mise en place de la communication et l'interaction plus réelles et mieux ancrées dans le contexte (inter)culturel. Du reste, la participation au projet créerait « un climat émotionnel favorable à l'apprentissage linguistique [tout en facilitant] le processus d'acquisition des savoirs » (Berdal-M asuy, Botella, 2013). En second lieu, les apprenants auraient l'occasion de s'impliquer dans une expérience authentique soutenant le développement de différentes compétences numériques - notamment celles relatives à la création des contenus, la coopération et la communication, mais également celles concernant la recherche d'information, la gestion et le traitement des données.

\subsection{L'apprentissage par problème dans l'environnement numérique}

La résolution de problème est une activité qui peut s'insérer à l'intérieur de plusieurs méthodes et approches pédagogiques. Néanmoins, nous l'évoquerons ici en tant que stratégie d'enseignement qui consiste à « regrouper les apprenants en équipe pour résoudre une situation-problème à la fois complexe et significative » (Vienneau, 2011 : 198) tout en offrant un cadre authentique afin de préserver le développement des compétences langagières et numériques.

Répandue dans la formation universitaire de différentes disciplines, comme les mathématiques, l'économie, l'informatique, les sciences de la santé, cette approche fournit des possibilités intéressantes pour l'enseignement des langues étrangères et notamment des langues de spécialité. À côté de la préparation des apprenants à leur future vie professionnelle, l'approche par problème :

« rompt avec la méthode traditionnelle car elle ne maintient pas l'étudiant dans un rôle passif de récepteur d'informations ; au contraire, elle sollicite sa participation active pour exposer sa vision du problème, pour défendre oralement ses idées, pour développer une argumentation solide, pour déployer ainsi sa créativité grâce à l'usage de l'instrument linguistique. » (Tano, 2016).

L'apprentissage par problème se construit autour d'une situation-problème (une activité visant à introduire une nouvelle connaissance) qui peut être classée selon « la tâche demandée, l'origine des problèmes, le mode de collecte 
des informations, l'engagement des apprenants [et] le support d'informations » (Guilbert, Ouellet, 2004 : 71). De ce fait, la tâche faisant appel au support d'informations numérique peut être considérée comme une résolution de problème à condition que l'apprenant soit exposé à une situation inconnue auparavant qui nécessite d'être maitrisée. L'environnement numérique, outre l'accès aux supports multimédias, propose des exploitations qui engageraient les apprenants non seulement sur le plan cognitif mais aussi affectif. À titre illustratif, les jeux sérieux (serious game), fondés sur une combinaison d'objectifs fictifs et réels, placeraient l'apprenant dans un processus de résolution de problème authentique ou quasi-authentique. Impliquant le jeu de rôle, l'interaction et la collaboration, les jeux sérieux, même s'ils restent concentrés sur l'aspect ludique (Grosbois, 2012 : 132), constituent un terrain d'exploitation intéressant pour l'apprentissage des langues. De plus, en fonction des objectifs de la tâche langagière imposée (authentique ou simulée), l'apprenant évoluerait dans un environnement numérique, utiliserait les technologies numériques de manière créative, résoudrait les problèmes techniques ou encore identifierait ses lacunes en compétences numériques (Carretero S., Vuorikari R., Punie Y., 2017 : 21).

En somme, la mise en place de l'apprentissage par problème dans l'environnement médiatisé offrirait des possibilités intéressantes pour combiner le développement des compétences numériques et langagières. D'un côté, la résolution de problème, s'effectuant par le biais d'une langue étrangère, créerait un contexte intéressant pour la production et l'interaction en langue étrangère fondées sur la participation active des apprenants. De l'autre côté, l'application de l'apprentissage par problème dans l'environnement médiatisé pourrait s'avérer propice pour l'engagement authentique de certaines habiletés numériques requises dans différents secteurs professionnels.

\section{Quelques réflexions finales}

Les compétences numériques deviennent de plus en plus indispensables dans la société contemporaine, c'est pourquoi l'éducation en matière de compétences numériques nécessite une approche plus globale et plus cohérente aussi bien à l'échelle mondiale que nationale. Le processus d'enseignementapprentissage des langues étrangères réalisé à distance et dans l'environnement médiatisé pourrait fournir des conditions nécessaires afin d'intégrer la mobilisation et le développement des compétences numériques. Néanmoins, pour que cette intégration soit réussie, il est nécessaire de mettre en place des activités fondées sur la construction active des connaissances et l'usage créatif du numérique en classe de langue. L'examen préliminaire des approches dites actives, à savoir l'apprentissage collaboratif, l'apprentissage par 
La classe de langue à l'ère du digital. Quelques réflexions sur l'intégration...

projet et l'apprentissage par problème, adaptées aux conditions de l'environnement médiatisé a montré que les approches en question offriraient un contexte intéressant pour développer les compétences langagières productives mais aussi celles relatives au fonctionnement dans le monde numérique. Toutefois, une analyse approfondie et basée sur la recherche empirique serait nécessaire afin d'en tirer des conclusions plus profondes. Tout bien considéré, la réflexion sur l'utilité des compétences numériques dans le processus d'enseignement-apprentissage des langues étrangères reste d'actualité. Étant donné que l'activité didactique demeure de plus en plus réalisée à distance et soutenue par différents dispositifs numériques, l'intégration des compétences numériques en didactique des langues étrangères semble nécessaire afin d'ajuster la formation linguistique à la mesure des défis du XXI ${ }^{\mathrm{e}}$ siècle.

\section{BIBLIOGRAPHIE}

Arpin L., Capra L. (2001), L'apprentissage par projets. Montréal/Chenelière : McGraw-Hill.

Bachy S. (2019), Comment se développe le savoir technopédagogique disciplinaire? « Spirale - Revue de recherches en éducation », n63, p. 125-137.

Berdal-Masuy F., Botella M . (2013), La pédagogie par le projet favorise-t-elle l'apprentissage linguistique? Mesure de l'impact émotionnel de ce type d'approche sur les apprenants. « Lidil », n 48. Online : http:// journals. openedition.org/lidil/3314 [consulté le 17.10.20].

Bouillon J.-L. (2015), Technologies numériques d'information et de communication et rationalisations organisationnelles : les « compétences numériques » face à la modélisation. « Les Enjeux de l'information et de la communication », $n^{\circ} 16 / 1$, p. 89-103. Online : https:// www.cairn.info/ revue-les-enjeux-de-l-information-et-de-lacommunication-2015-1-pag e-89.htm [consulté le 5.07.2020].

BuchholtzS. et al. (2015), Analiza doświadczeń oraz identyfikacja dobrych praktyk w obszarze wspierania rozwoju kompetencji cyfrowych w kontekście przygotowania szczegółowych zasad wdrażania Programu Operacyjnego Polska Cyfrowa na lata 2014-2020 oraz koordynacji celu tematycznego. Raport Końcowy, Warszawa. Online : https://www.polskacyfrowa.gov.pl/med ia/5180/RK_kompetencje_cyfrowe.pdf [consulté le 8.07.2020].

Budzisz W. et al. (2014), Katalog kompetencji medialnych, informacyjnych i cyfrowych. Warszawa: Fundacja Nowoczesna Polska. Online: http://eduk acjamedialna.edu.pl/media/chunks/attachment/Katalog_kompetencji _medialnych_2014_EudBrrl.pdf [consulté le 8.07.2020]. 
Carretero S., Vuorikari R., Punie Y. (2017), DigComp 2.1. The Digital Competence Framework for Citizens. Luxembourg: Publications Office of the European Union. Online : https://ec.europa.eu/jrc/en/publication/eur-scientific-and -technical-research-reports/digcomp-21-digital-competence-framework-c itizens-eight-proficiency-levels-and-examples-use [consulté le 10.07.2020].

Commission européenne (2006), RECOM M ANDATION DU PARLEM ENT EUROPÉEN ET DU CONSEIL du 18 décembre 2006 sur les compétences clés pour l'éducation et la formation tout au long de la vie. Online : https:// eur-lex.europa.eu/legal-content/FR/TXT/?uri=celex:32006H0962 [consulté le 10.07.2020].

Commission européenne (2018), RECOM M ANDATION DU CONSEIL du 22 mai 2018 relative aux compétences clés pour l'éducation et la formation tout au long de la vie. Online : https://op.europa.eu/pl/publication-det ail/-/publication/6fda126a-67c9-11e8-ab9c-01aa75ed71a1/language-f r/format-PDF [consulté le 10.07.2020].

Commission européenne (2012), Manifeste pour les compétences numériques. Bruxelles, European Schoolnet.

Coulet J.-C. (2011), La notion de compétence : un modèle pour décrire, évaluer et développer les compétences. "Le travail humain », vol. 74, p. 1-30. Online : https:// www.cairn.info/ revue-le-travail-humain-2011-1-page-1. htm [consulté le 9.07.2020].

Dauphin F. (2012), Culture et pratiques numériques juvéniles : Quels usages pour quelles compétences? «Questions vives», Vol.7, $n^{\circ} 17$ : Des usages des TIC à la certification des compétences numériques : quels processus de formation et de validation ? Online : https://journals.openedition.org /questionsvives/988 [consulté le 14.09.2020].

Filiciak M., Mazurek P., Growiec K. (2013), Korzystanie z mediów a podziały społeczne. Warszawa : Centrum Cyfrowe Projekt : Polska,

Glikman V. (2002), Des cours par correspondance au "e-learning”. Paris : Presses Universitaires de France.

Grosbois M. (2012), Didactique de langues et technologies. Paris : Presses de I'université Paris-Sorbonne.

Guilbert L., Ouellet L. (2004), Étude de cas - Apprentissage par problèmes. Sainte-Foy : Presses de l'Université du Québec.

Henri F. (2010), « Collaboration, communautés et réseaux: partenariats pour l'apprentissage. In : Charlier B., Henri F. (dir.), Apprendre avec les technologies. Paris : Presses Universitaires de France, p. 169-180.

Henri F., Lundgren-Cayrol K. (2001), Apprentissage collaboratif à distance. Sainte-Foy : Presses de l'Université du Québec. 
La classe de langue à l'ère du digital. Quelques réflexions sur l'intégration...

Jasiewicz J. et al. (2015), Ramowy katalog kompetencji cyfrowych. Online: https:// mc.bip.gov.pl/rok-2015/ramowy-katalog-kompetencji-cyfrowych.html [consulté le 7.07.2020].

Lebrun M., Lison Ch., Batier Ch. (2016), Les effets de l'accompagnement technopédagogique des enseignants sur leurs options pédagogiques, leurs pratiques et leur développement professionnel. « Revue internationale de pédagogie de l'enseignement supérieur », n 32-1. Online : http:// ripes.revues.org/1028 [consulté le 2.09.2020].

Mangenot F. (2011), Introduction. Du e-learning aux interactions pédagogiques en ligne. In : Nissen, E. et al. (dir.), Interagir et apprendre en ligne. Grenoble : ELLUG Université Stendhal, p. 7-20.

M angenot F., Soubrié T. (2010), Classer des cybertâches : quels critères ? quels obstacles ? «Éla. Études de linguistique appliquée », nº 160. p. 433-443. Mell L. (2018), À travers les hypostases du numérique dans l'enseignement supérieur : réflexion critique sur le développement de la culture et des compétences numériques. « Lien social et Politiques », n 81, p. 173-191. Online : https:// www.erudit.org/fr/revues/lsp/2018-n81-Isp04317/10 56310ar/ [consulté le 2.09.2020].

M inistère de l'Education nationale et de la Jeunesse (2019), Document d'accompagnement - mise en œuvre du cadre de référence des compétences numériques (CRCN). Online : https://cache.media.eduscol.education.fr/ file/CRCNum/57/0/Document_accompagnement_CRCN_1205570.pdf [consulté le 17.09.2020].

Nissen E. (2019), Formation hybride en langues - Articuler présentiel et distanciel. Paris : Didier.

Nowicka M., Dziekońska J. (red.), (2018), Cyfrowy tubylec w szkole, diagnozy i otwarcia. Tom I: Współczesny uczeń a dydaktyka 2.0. Toruń: Wydawnictwo Adam M arszałek.

Ollivier Ch., Puren L. (2011), Le Web 2.0 en classe de langue. Une réflexion théorique et des activités pratiques pour faire le point. Paris : Éditions $M$ aison des Langues.

Plebańska M., Szyller A., Sieńczewska M. (2020), Q edukacji cyfrowej. Warszawa: Wydawnictwo Difin.

Prensky M . (2001), Digital Natives, Digital Immigrants. On the Horizon, Vol.9, no 5. MCB University Press. Online : https://www.marcprensky.com/writ ing/Prensky\%20-\%20Digital\%20Natives,\%20Digital\%20lmmigrants\%20-\% 20Part1.pdf [consulté le 3.09.2020].

Proulx J. (2004), L'apprentissage par projet. Québec : Presses Universitaires de l'Université du Québec. 
Puentedura R. (2006), Transformation, technology, and education. Online : http:// hippasus.com/resources/tte/ [consulté le 7.07.2020].

Rossenberg M. J. (2000), E-Learning: Strategies for Delivering Knowledge in the Digital Age. McGraw-Hill Education, New York.

Tano M. (2016), L'apprentissage par problèmes : une méthode active d'enseignement des langues étrangères pour spécialistes d'autres disciplines. « Recherche et pratiques pédagogiques en langues de spécialité », Vol. 35, $\mathrm{n}^{\circ}$ spécial 1. Online : http://journals.openedition.org/apliut/5553 [consulté le 15.10.2020].

Vienneau R. (2011), Apprentissages et enseignements. Théories et pratiques. Montréal : Gaëtan M orin Éditeur.

Walckiers M . \& De Praetere T., 2004 : L'apprentissage collaboratif en ligne, huit avantages qui font un must. « Distances et savoirs», Vol. 2, p. 53-75.

Żylińska, M. (2013), Neurodydaktyka. Nauczanie i uczenie się przyjazne mózgowi. Toruń: Wydawnictwo UMK.

Received: 30.10 .2020

Revised: 14.05.2021 